\title{
As perturbações emocionais - ansiedade e depressão na criança e no adolescente
}

Margarida Crujo, * Cristina Marques**

\section{RESUMO}

A Perturbação da Ansiedade e a Perturbação Depressiva constituem entidades clínicas de elevada prevalência na infância e na adolescência, e podem ser precursoras de psicopatologia na idade adulta. Por ambos os motivos, o seu diagnóstico precoce e intervenção atempada tornam-se essenciais, assim como a detecção de eventuais factores de risco que as possam promover. Os Cuidados de Saúde Primários assumem, neste contexto, uma função primordial na triagem de casos, assim como na intervenção e eventual referenciação para os serviços de Pedopsiquiatria.

Neste artigo, abordamos, abreviadamente, aspectos referentes aos factores de risco, prevalência, diagnóstico, intervenção clínica e prognóstico para as Perturbações da Ansiedade e Depressiva.

Palavras-chave: Ansiedade, depressão, criança, adolescente.

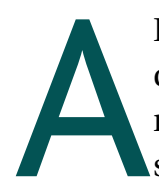

Perturbação da Ansiedade (PA) e a Perturbação Depressiva (PD) são entidades comuns na criança e no adolescente. A primeira apresenta uma prevalência estimada entre os $4 \mathrm{e}$ os $20 \%,{ }^{1}$ enquanto que a outra entre 1 e $2 \%$ em idade pré-pubertária, e 3 a 8\% se nos referirmos à adolescência. $^{1}$

Vamos, então, deter-nos em cada uma das perturbações individualmente - primeiro a da ansiedade, posteriormente a depressiva - e tentar realçar os aspectos que nos parecem mais importantes no contexto dos Cuidados de Saúde Primários.

\section{PERTURBAÇÕES DA ANSIEDADE}

\section{Aspectos Gerais}

Em psicopatologia, tal como em Medicina, no geral, é determinante a distinção entre o normal e o patológico. Quer o medo, quer a ansiedade, podem constituir, antes de mais, reacções normais a situações adversas ou de perigo: se o medo permite ao indivíduo afastar-se de um risco objectivo, a ansiedade assume o mes-

*Interna de Psiquiatria da Infância e da Adolescência do Hospital de Dona Estefânia - Centro Hospitalar de Lisboa Central.

**Psiquiatra da Infância e da Adolescência do Hospital de Dona Estefânia Centro Hospitalar de Lisboa Central. Assessora da Coordenação Nacional para a Saúde Mental. mo papel protector, ainda que esta surja na ausência de um estímulo ameaçador e se coloque no campo da antecipação. No entanto, quando ambas deixam de ser adaptativas, podemos ter que passar a falar em sintomas e/ ou perturbações da ansiedade.

Se brevemente atravessarmos as faixas etárias que este artigo abrange, podemos assumir que é normal os bebés reagirem com medo perante o escuro ou sons que desconhecem e, também, a partir de determinada altura - aproximadamente aos 6 meses, sem que seja possível sermos exactos -, a rostos não familiares ou ao afastamento da(s) figura(s) de referência. É normal que crianças em idade pré-escolar apresentem receios relativos a animais, a criaturas imaginárias, como monstros e fantasmas, à rejeição, a trovoadas e ruídos, também ao escuro. ${ }^{2,3}$ Assim como é normal que crianças, já em idade escolar, refiram grandes preocupações com as amizades e com a saúde física, também os adolescentes se podem centralizar em temas mais abstractos, como a morte, a religião, questões sociais e também sexuais. $^{3}$

Apesar disto, a distinção entre o ser normal e o ser patológico tem que ocorrer na prática clínica.

Os comportamentos da criança/adolescente devem ser avaliados a fim de detectarmos se os sintomas in- 
vadem a sua rotina, o que se pode manifestar por padrões mais desajustados, como o evitamento perante actividades adequadas ao sexo e à idade. Há também que efectuar uma pesquisa de sintomatologia somática; são exemplos as palpitações, as cefaleias, os vómitos, a hipersudorese. A duração dos sintomas deve ser determinada, bem como o enquadramento dos mesmos no desenvolvimento psicossocial da criança e do adolescente.

Os medos/receios ditos normais das diversas faixas etárias são transitórios e pouco intensos, e não têm repercussões, nem no desenvolvimento, nem na rotina da criança ou do adolescente.

\section{FACTORES DE RISCO E PREVALÊNCIA}

As PA são o segundo grupo mais comum de perturbações psiquiátricas em idade pediátrica, imediatamente a seguir às perturbações do comportamento, e com uma prevalência superior às perturbações depressivas ou às perturbações de hiperactividade e défice de atenção. ${ }^{2}$

Do ponto de vista da etiologia, parecem estar implicados factores constitucionais (genéticos, do temperamento) e sócio-familiares, nomeadamente padrões desadequados de funcionamento familiar. São de destacar alguns dados referidos na literatura:

- existe uma maior probabilidade de surgirem PA em filhos de pais que apresentem PA; ${ }^{2}$

- crianças de temperamento inibido e tímido parecem estar em maior risco de posteriormente virem a desenvolver PA; ${ }^{2,3}$

- acontecimentos de vida adversos podem condicionar o aparecimento de psicopatologia e possuem um carácter cumulativo;

- em termos de funcionamento familiar, a transmissão da patologia pode processar-se por aprendizagem e modelagem, ${ }^{2}$ ou por incapacidade dos cuidadores em reduzirem reacções de medo. ${ }^{3}$

Neste último ponto, especificamente, os Cuidados Primários podem ter uma função primordial no tratamento e/ou referenciação dos pais.

A maior prevalência de PA ocorre no género feminino $^{1,3} \mathrm{e}$, habitualmente, em idade pré-pubertária. ${ }^{1}$ Não são consensuais os valores desta preponderância, talvez porque o estudo das PA ainda é recente, se comparado com o de outras perturbações psiquiátricas. ${ }^{1} \mathrm{Em}$ períodos de transição na escola, como na passagem da pré-primária para o Primeiro Ciclo, e entre ciclos, a prevalência da ansiedade também aumenta. ${ }^{4}$

\section{Classificação e Comorbilidades}

As PA podem ser classificadas em: Perturbação de Ansiedade de Separação, Perturbação de Ansiedade Social/Fobia Social, Fobias Específicas, Perturbação de Ansiedade Generalizada e Perturbação de Pânico, com ou sem Agorafobia. Passaremos a resumir cada uma delas.

A ansiedade perante a separação do cuidador ocorre normalmente em crianças entre os seis meses e os três anos, diminuindo a partir desta idade. No entanto, assume contornos patológicos quando surge numa idade não usual - especialmente a partir dos seis anos $-^{1}$ ou quando as manifestações de ansiedade são demasiado intensas perante ou na antecipação da separação. São manifestações uma preocupação excessiva acerca da eventualidade de perda da figura de referência, queixas somáticas repetidas, recusa em estar sozinho ou em ir dormir, ocorrência de pesadelos. Pode constituir um factor de risco para perturbação de pânico ou agorafobia na idade adulta. ${ }^{1}$

A Fobia Social consiste na sensação de medo persistente perante a exposição a situações sociais ou de avaliação, tais como o falar em público ou comunicar com estranhos. Quem apresenta a perturbação tende a evitar as situações que o angustiam, ou vive-as com uma ansiedade imensa, que se pode manifestar por um aumento da frequência cardíaca, tonturas, rubor, alterações do trânsito gastrointestinal. O temperamento inibido em crianças pode ser preditor desta patologia. ${ }^{1}$

Existem outras fobias, que se traduzem por um medo intenso de objectos ou situações específicas: de andar de avião, de animais, das alturas, de ver sangue.

A Perturbação de Ansiedade Generalizada ocorre mais frequentemente na adolescência, e manifesta-se por preocupações excessivas, incontroláveis e múltiplas. Estas preocupações habitualmente centram-se em temas relacionados com competência, acontecimentos futuros e desconhecidos, aprovação, ${ }^{1}$ catástrofes, pontualidade. São comuns sintomas como irritabilidade, fadiga, dificuldades de concentração e queixas somáticas. Pais muito exigentes ou, pelo contrário, demasiado flexíveis ou permissivos constituem um factor 
de risco para este tipo de perturbação.

A Perturbação de Pânico, por sua vez, caracteriza-se por crises inesperadas de ansiedade, acompanhadas por sintomas somáticos e outros cognitivos, tais como medo de morrer, de enlouquecer, de perder o controlo. O pico da prevalência ocorre entre os 15 e os 19 anos de idade. A Agorafobia é diagnosticada se o indivíduo evita locais dos quais sinta que não possa sair facilmente.

Podemos ainda falar um pouco sobre outro diagnóstico que está relacionado com questões de ansiedade, nomeadamente a ansiedade de separação ou ansiedade de desempenho: a Recusa Escolar. Surge mais frequentemente nos períodos de transição escolar, e traduz-se por uma angústia intensa, receio de ficar só, comportamentos de dependência, alterações do sono, medos e queixas somáticas. As crianças que recusam ir à escola manifestam um desejo imperioso de permanecer em casa, mantêm o interesse pelos estudos ${ }^{5} \mathrm{e}$ têm habitualmente boa capacidade de aprendizagem e de desempenho escolar. ${ }^{4}$

As PA estão frequentemente associadas a situações comórbidas - $50 \%$ dos casos, sendo o mais comum a coocorrência de outra perturbação da ansiedade e, imediatamente a seguir, de perturbações depressivas. ${ }^{1} \mathrm{~A}$ questão das comorbilidades torna-se muito relevante, principalmente se atendermos ao facto de provocarem um impacto ainda maior na vida da criança ou do adolescente.

\section{Intervenção Terapêutica}

Os pais devem ser aconselhados a tranquilizar a criança, mantendo uma atitude firme e segura; a criança/adolescente deve ser incentivada a enfrentar o problema e a procurar uma solução para o mesmo; não se deve favorecer o evitamento excessivo das situações que causam ansiedade, sem que, no entanto, a criança/adolescente seja forçada para além da sua capacidade de adaptação. ${ }^{6} \mathrm{O}$ tratamento de $\mathrm{PA}$ ou PD nos pais é algo também a ter em consideração.

Para além das medidas de intervenção mencionadas, a criança/adolescente tem indicação para um seguimento psicoterapêutico. O tratamento farmacológico, por seu turno, pode ser um coadjuvante da abordagem anterior. Ainda que a literatura seja pouco consensual relativamente ao uso de benzodiazepinas - devido ao risco de habituação e aos seus eventuais efeitos paradoxais -, estas podem ser ponderadas, de forma transitória, em situações agudas pontuais. Os antidepressivos podem constituir uma alternativa, assim como antipsicóticos em doses baixas. São utilizados, na prática clínica, em situações de alguma gravidade e quando existem situações de comorbilidade, embora a sua ponderação deva ser feita pelo especialista.

\section{Prognóstico}

Em relação à evolução a longo termo, as PA apresentam, geralmente, um prognóstico relativamente bom. ${ }^{3,7}$ Ainda assim, estudos prospectivos apontam para uma continuidade entre uma percentagem minoritária de PA na idade pediátrica - especialmente a Perturbação de Ansiedade Generalizada $-{ }^{1}$ e PA ou PD na vida adulta. ${ }^{2,7}$

\section{PERTURBAÇÕES DEPRESSIVAS}

\section{Aspectos Gerais}

A depressão infantil foi reconhecida tardiamente, e apenas a partir dos Anos 60 do século passado a perturbação foi incluída num sistema de classificação, criado pelo Group for the Advancement of Psychiatry (1966). Esta classificação enfatizou o facto de a depressão nesta faixa etária apresentar diferenças relativamente aos quadros depressivos dos adultos. Até então, acreditava-se que os pré-adolescentes eram incapazes de experienciar depressões, e os quadros depressivos que surgiam nos adolescentes eram desvalorizados e justificados como uma crise própria da idade. ${ }^{8}$ Talvez para tal tenha contribuído o facto de as crianças terem mais dificuldade em expressar plenamente os seus afectos. Hoje em dia sabemos, pois, que tal não sucede desta maneira e que, existindo queixas verbalizadas ou apenas comportamentos observados, a depressão pode existir em todas as faixas etárias.

\section{Factores de Risco}

Existem factores de risco individuais, familiares e sociais ou do meio.

Nos factores individuais, destacamos os aspectos genéticos, o temperamento, a capacidade de regulação emocional, o estilo cognitivo, a personalidade. Por exemplo, crianças/adolescentes com dificuldades de adaptação a mudanças têm maior tendência para a depressão; crianças/adolescentes deprimidos tendem 
a atribuir a ocorrência de acontecimentos negativos a características do próprio. ${ }^{9}$

Pela sua relevância, gostaríamos de salientar os fac-

\section{tores familiares.}

Nas famílias de crianças e adolescentes com depressão são frequentemente encontrados padrões interactivos nos quais predominam atitudes de rejeição e hostilidade, ou de intrusividade e sobre-envolvimento. As falhas no suporte afectivo e na coesão familiar estão habitualmente presentes, impregnando um modelo de funcionamento frequentemente conflitual entre os vários elementos do sistema familiar.

Os padrões de sobre-envolvimento são os mais difíceis de detectar, já que se encontram, não raras vezes, "camuflados» por atitudes de aparente preocupação/hiperprotecção. Nestes casos as relações costumam girar em torno de atitudes desnarcisantes (de rejeição e hostilidade) e de uma exigência extrema da mãe ou do pai, nem sempre evidentes aos olhos dos outros e do próprio filho.

A meta imposta é inatingível, o filho, apesar de todos os seus esforços, acaba por soçobrar. Nestas famílias é difícil para a criança definir a sua identidade; mais fácil será submeter-se, anular-se, adaptar-se e, face ao esgotamento dos mecanismos de adaptação, deprimir-se.

A patologia depressiva dos pais é outro factor de risco importante. De facto, diversos mecanismos têm vindo a ser propostos como modelos explicativos para o desenvolvimento de perturbações afectivas nos filhos de pais deprimidos.

Embora os factores genéticos aumentem o risco de depressão, é a interacção entre factores genéticos e do meio envolvente que se revela crítica para o aparecimento de formas mais graves de depressão na criança e no adolescente. ${ }^{9}$ São, em particular, os padrões relacionais que se estabelecem nestas famílias que medeiam o impacto que a doença depressiva dos pais tem na criança.

Nos pais com patologia depressiva podem predominar os estilos interactivos atrás referidos, quer no sentido da falha no suporte relacional, quer no sentido da intrusividade, da exigência narcísica.

Finalmente, nos factores sociais, destacamos as mudanças escolares, a percepção dos outros como críticos ou rejeitantes, ${ }^{9}$ as dificuldades na socialização.

Os factores de risco podem assumir um carácter cró- nico (como os maus-tratos ou a exposição a conflitos familiares permanentes), ou agudo, como luto e outras situações de perda. Há, no entanto, uma grande variabilidade nas respostas de cada criança face a estes últimos e, de entre todos estes factores, as experiências de adversidade crónica parecem ser as de maior relevo no desenvolvimento futuro de psicopatologia.

\section{Apresentação Clínica e Prevalência}

A noção de depressão é clínica e, neste âmbito, o termo depressão refere-se a uma doença caracterizada pela presença de uma alteração do humor persistente e suficientemente grave para ser considerada «perturbação".

Frequentemente, o humor depressivo está inserido numa constelação de outros sintomas. Constituem exemplos a anedonia (incapacidade de sentir prazer), a culpa inapropriada, a baixa da auto-estima e a desesperança, o cansaço ou a perda de energia, a diminuição da concentração ou da capacidade de pensar, a agitação ou a lentificação psicomotora, as alterações do sono e do apetite, os pensamentos mórbidos ou suicidários. ${ }^{10}$

Esta constelação de sintomas enunciada, ainda assim, pode não constituir, nem um episódio depressivo, nem uma perturbação depressiva, sendo determinante uma vigilância estreita da criança ou do adolescente que a apresente. O que determinará a gravidade da situação clínica e, consequentemente a decisão de estarmos perante um sintoma depressivo, um conjunto de sintomas depressivos - ou síndrome -, ou uma perturbação depressiva, serão factores como a persistência da sintomatologia, o mal-estar clinicamente significativo, o impacto na rotina da criança ou do adolescente, qualquer que seja a área de funcionamento. Esta apreciação terá, pois, implicações na definição da estratégia clínica.

O quadro clínico da depressão pode assumir características mais específicas de acordo com a faixa etária.

Embora os quadros depressivos de adolescentes e adultos apresentem muitas semelhanças, o mesmo não acontece com crianças mais novas, tornando-se as diferenças ainda mais marcadas na idade pré-escolar. Estas diferenças reflectem, provavelmente, as dificuldades que as crianças mais novas apresentam, devido às suas competências cognitivas e de linguagem, em dis- 
tinguir e verbalizar emoções e cognições negativas, como a desesperança, a desvalorização ou a culpabilidade. ${ }^{11}$

Os bebés podem apresentar apatia, grande irritabilidade, insónia, choro intenso e aparentemente não motivado, recusa em comer.

$\mathrm{Na}$ idade pré-escolar, sintomas de distimia estão, com frequência, clara e indiscutivelmente presentes, como humor irritável, alterações do sono e do apetite. Para além destes, surgem também comportamentos de franca oposição, com baixa tolerância a situações de frustração, bem como dificuldades ao nível da autonomia e da separação do adulto do qual dependem.

Já na idade escolar e na pré-adolescência, pode surgir a verbalização de sentimentos depressivos (culpa, desesperança, negativismo), embora a irritabilidade seja mais frequente que o humor depressivo. Podem também ocorrer o isolamento social, dificuldades escolares com diminuição da concentração e sintomas somáticos, como as cefaleias e as epigastralgias.

Não raramente, o quadro clínico pode assumir características de uma perturbação do comportamento - incapacidade de lidar com a frustração, agressividade, impulsividade. No entanto, nestes casos, a presença de uma alteração do humor (depressivo ou irritável) permite fazer o diagnóstico diferencial com as perturbações do comportamento propriamente ditas. É de salientar que o humor depressivo não é frequentemente percepcionado pelos pais, sendo até negado quando os questionamos sobre este aspecto; em muitas situações, é a entrevista com a criança (e o que esta nos faz sentir) que nos leva a colocar este diagnóstico. Este facto vem reforçar a absoluta necessidade de não só observarmos individualmente a criança, como de estarmos atentos ao nosso estado emocional, para uma correcta avaliação clínica.

Durante a adolescência, por seu turno, podem surgir preocupações com o próprio corpo, nomeadamente com os caracteres sexuais secundários, o acne, o peso. Por comparação com a sintomatologia da idade escolar, as alterações do sono e do apetite podem tornar-se mais preponderantes, assim como os sentimentos de desesperança, a anedonia e a ideação suicida. A prevalência das queixas somáticas e de ansiedade, por seu turno, diminui. ${ }^{3}$ Principalmente na adolescência, a avaliação do risco de suicídio, mesmo se a priori este não for evidente, tem obrigatoriamente que se realizar; as tentativas de suicídio, neste grupo etário, podem assumir contornos de grande letalidade.

Se até à adolescência, a prevalência de PD é idêntica nos dois géneros, a partir desta faixa etária o género feminino apresenta uma prevalência duas a três vezes superior ao masculino. Factores hormonais podem contribuir para esta diferença. ${ }^{3,12}$

\section{Classificação e Comorbilidades}

As Perturbações Depressivas podem ser encaradas como pertencendo a um continuum, que vai desde as Perturbações de Adaptação, às Perturbações Depressivas Major, passando pelas Distimias.

Sucintamente e de uma forma simples, nas Perturbações de Adaptação existe um factor de stress identificado, e os sintomas depressivos surgem até três meses após o contacto com esse factor, deixando de existir, posteriormente, em seis meses ou menos.

Nas Distimias, por sua vez, a sintomatologia é mais ligeira, se comparada com a das perturbações major, embora crónica; portanto, o humor depressivo ou irritável (principalmente nas crianças) ocorre durante a maior parte do dia, e durante quase todos os dias por um período de pelo menos um ano. Outros dois sintomas, no mínimo, devem estar presentes, de entre: alterações do apetite e do sono, cansaço ou perda de energia, dificuldade na concentração ou no poder de decisão, baixa auto-estima, e sentimentos de desesperança.

O Episódio Depressivo Major contempla, obrigatoriamente, o humor depressivo ou irritável - também presente durante a maior parte do dia e quase todos os dias durante pelo menos duas semanas -, ou a perda de interesse ou prazer (anedonia) em actividades previamente investidas com satisfação. Para além destes, pelo menos cinco dos seguintes devem existir: alterações do peso e do sono, agitação ou lentificação psicomotora, cansaço ou perda de energia, diminuição na capacidade de pensar ou de se concentrar, sentimentos de desvalorização ou de culpa inapropriada, e ideações de morte ou suicida. Chamamos Perturbação Depressiva Major quando, ao longo da evolução clínica de um indivíduo, ocorre pelo menos um episódio depressivo major. $^{13}$

As Perturbações Bipolares, ainda que, tal como as Perturbações Depressivas e as Perturbações da Ansie- 
dade, sejam consideradas Perturbações Emocionais, não são aqui mencionadas por nos parecer que não se enquadram no âmbito do artigo; o seu diagnóstico diferencial é difícil, pelo que a avaliação deve ser feita pelo Pedopsiquiatra.

Nas Perturbações Depressivas, mais de 50\% dos casos apresentam comorbilidades. As mais frequentes são as Perturbações do Comportamento - cerca de $40 \%$ das crianças/adolescentes com depressão -, seguidas das Perturbações da Ansiedade, que ocorrem em aproximadamente $34 \%$ dos casos. ${ }^{8}$

Há, de facto, uma forte associação entre ansiedade de separação e depressão em crianças e adolescentes, independentemente da idade e do género. ${ }^{11}$ Numerosos estudos demonstraram que elevados níveis de ansiedade são frequentemente preditores do aparecimento subsequente de depressão, ${ }^{11}$ colocando a hipótese de a ansiedade representar um precursor da depressão e que o aparecimento de sintomas de depressão causaria uma diminuição dos sintomas de ansiedade, como se houvesse uma "transformação" da ansiedade em depressão.

\section{Intervenção Terapêutica}

A intervenção terapêutica deve corresponder à gravidade do quadro clínico, sendo imprescindível a exclusão do risco de suicídio, como já referimos.

Para além de mostrar disponibilidade para ouvir as preocupações da criança ou do adolescente, o clínico deve intervir na família - sensibilização dos pais para o sofrimento da criança/adolescente e discussão de estratégias para lidar com o problema-, escola-integração no grupo de pares, por exemplo - e rede social de apoio - integração em projectos locais de intervenção sócio-cultural, tal como Escuteiros, ATL e outras actividades organizadas. ${ }^{14}$

Nunca é demais enfatizar a importância que uma atitude de escuta empática pode ter na boa resolução deste tipo de situações.

Concomitantemente, devem ainda ser implementadas intervenções psicoterapêuticas e, nos casos de maior gravidade, intervenções psicofarmacológicas com antidepressivos, que complementem as primeiras. O uso de antidepressivos nas faixas etárias a que nos temos vindo a reportar não é consensual, pois a evidência dos benefícios da sua administração nos ensaios clínicos também não o é - e menos ainda em crianças e pré-adolescentes. No entanto, na prática clínica estes fármacos são habitualmente utilizados na adolescência, com respostas terapêuticas positivas.

\section{Prognóstico}

Aproximadamente 2/3 das crianças ou adolescentes com Perturbações Depressivas melhora significativamente, ${ }^{3}$ mesmo que a recuperação completa se prolongue por meses ou até anos. No entanto, factores como a gravidade da sintomatologia e a presença de comorbilidades influenciam negativamente o prognóstico. As crianças com Distimia têm maior probabilidade de apresentar episódios depressivos major no futuro, ainda que os episódios depressivos nesta faixa etária tenham menor probabilidade de recorrer posteriormente. Na adolescência, perturbações de gravidade moderada a severa têm maior risco de suicídio ou de dificuldades relacionais persistentes na idade adulta; a recorrência de episódios depressivos Major é comum.

Terminamos com um extracto de um livro de Saramago: «(...) Aquele rosto nu, sem óculos, com o bigode ligeiramente crescido, pêlo e cabelo têm vida mais longa, exprimia uma grande tristeza, daquelas sem emenda, como as da infância, que, por da infância serem, julgamos terem remédio fácil, esse é o nosso engano (...)».15

Os autores declararam não possuir conflitos de interesses.

\section{ENDEREÇO PARA CORRESPONDÊNCIA \\ Margarida Crujo \\ E-mail: mmcrujo@hotmail.com \\ Cristina Marques \\ E-mail: cristina.marq@hotmail.com}

\section{REFERÊNCIAS BIBLIOGRÁFICAS}

1. Krain AL, Ghaffari M, Freeman J, Garcia A, Leonard H, Pine DS. Anxiety Disorders. In: Martin A, Volkmar FR, editors. Lewis's Child and Adolescent Psychiatry: A Comprehensive Textbook. 4th ed. Philadelphia: Lippincott Williams \& Wilkins; 2007. p. 538-47.

2. Goodman R, Scott S. Anxiety disorders. In: Goodman R, Scott S, editors. Child Psychiatry. 2nd ed. Oxford: Blackwell Publishing; 2005. p. 84-90 .

3. Turk J, Graham P, Verhulst F. Emotional development and disorders of mood. In:Turk J, Graham P, Verhulst F, editors. Child and adolescent psychiatry: a developmental approach. 4th ed. New York: Oxford University Press; 2007. p. 221-47.

(4) Tonge BJ. Common child and adolescent psychiatric problems and their management in the community. MGA Practice Essentials. Disponível 
em:http://www.mja.com.au/public/mentalhealth/articles/tonge/tonge. html\#suba55 [acedido em 08/10/2009].

4. Marques C, Cepêda T. Recusa Escolar. In: Coordenação Nacional para a Saúde Mental, editor. Recomendações para a prática clínica da saúde mental infantil e juvenil nos Cuidados de Saúde Primários. Lisboa: Coordenação Nacional para a Saúde Mental; 2009. p. 31-32.

5. Marques C, Cepêda T. Perturbações da ansiedade. In: Coordenação Nacional para a Saúde Mental, editor. Recomendações para a prática clínica da saúde mental infantil e juvenil nos Cuidados de Saúde Primários. Lisboa: Coordenação Nacional para a Saúde Mental; 2009. p. 28-29 .

6. Klein RG, Pine DS. Anxiety Disorders. In: Rutter M, Taylor E, editors. Child and Adolescent Psychiatry. 4th ed. Oxford: Blackwell Publishing; 2002. p. 483-509.

7. Harrington R. Affective disorders. In: Rutter M, Taylor E, editors. Child and adolescent psychiatry. 4th ed. Oxford: Blackwell Publishing; 2002. p. 463-82.

8. McCauley E, Pavlidis K, Kendall K. Developmental precursors of depression: the child and the social environment. In: Goodyer IM, editor. The Depressed Child and Adolescent. 2nd ed. Cambridge: Cambridge University Press; 2001. p. 46-78.
9. Goodman R, Scott S. Depression and Mania. In: Goodman R, Scott S, editors. Child Psychiatry. 2nd ed. Oxford: Blackwell Publishing; 2005. p. 91-96.

10. Kolvin I, Sadowski H. Childhood depression: clinical phenomenology and classification. In: Goodyer IM, editor. The Depressed Child and Adolescent. 2nd ed. Cambridge: Cambridge University Press; 2001. p. 119-42.

11. Brent DA, Weersing VR. Depressive disorders. In: Martin A, Volkmar FR, editors. Lewis's Child and Adolescent Psychiatry: a Comprehensive Textbook. 4th ed. Philadelphia: Lippincott Williams \& Wilkins; 2007. p. 503-513.

12. American Psychiatric Association. DSM-IV - Diagnostic and Statistical Manual of Mental Disorders. 4th ed. Washington, DC:American Psychiatric Association Press; 1994.

13. Marques C, Cepêda T. Perturbações do Humor. In: Coordenação Nacional para a Saúde Mental, editor. Recomendações para a prática clínica da saúde mental infantil e juvenil nos Cuidados de Saúde Primários. Lisboa: Coordenação Nacional para a Saúde Mental; 2009. p. 29-31.

14. Saramago J. $O$ ano da morte de Ricardo Reis. $6^{a}$ ed. Lisboa: Editorial Caminho; 1984.

\section{ABSTRACT}

\section{EMOTIONAL DISORDERS - ANXIETY AND DEPRESSION IN CHILDHOOD AND ADOLESCENCE}

Anxiety Disorder and Depressive Disorder are clinical entities of high prevalence in childhood and adolescence, and may be precursors of psychopathology in adulthood. For both reasons, early diagnosis and intervention become essential, as well as the identification of possible risk factors that enhance these disorders. Primary Health Care assumes, in this context, an important role in screening cases, as well as in intervention and possible referral to Child and Adolescent Psychiatry Services. In this article the authors briefly discuss aspects related to risk factors, prevalence, diagnosis, clinical intervention and prognosis for Anxiety Disorder and Depressive Disorder.

Keywords: Anxiety; Depression; Child; Adolescent. 